\title{
Multiplicity of solutions for elliptic problems of $p$-Kirchhoff type with critical exponent
}

\author{
Chenxing Zhou' and Yueqiang Song ${ }^{2 *}$
}

\section{"Correspondence:}

songyueqiang@sohu.com

${ }^{2}$ Scientific Research Department,

Changchun Normal University,

Changchun, Jilin 130032, P.R. China

Full list of author information is

available at the end of the article

\section{照 Springer}

\begin{abstract}
In this paper we consider a class of elliptic problems of $p$-Kirchhoff type with critical exponent in bounded domains and new results as regards the existence and multiplicity of solutions are obtained by using the concentration-compactness principle and variational method.
\end{abstract}

MSC: $35 \mathrm{~J} 70 ; 35 \mathrm{~B} 20$

Keywords: $p$-Kirchhoff-type problem; critical growth; concentration-compactness principle; variational method

\section{Introduction}

In this paper we deal with the existence and multiplicity of solutions to the following $p$-Kirchhoff type with critical exponent:

$$
\left\{\begin{array}{l}
-\left[g\left(\int_{\Omega}|\nabla u|^{p} d x\right)\right] \Delta_{p} u=\lambda h(x, u)+u^{p^{*}-2} u, \quad x \in \Omega, \\
u=0, \quad x \in \partial \Omega,
\end{array}\right.
$$

where $1<p<N, \lambda$ is a positive parameter, $\Omega \subset \mathbb{R}^{N}$ is an open bounded domain with smooth boundary and $\lambda$ is a positive parameter, $p^{*}=N p /(N-p)$ is the critical exponent according to the Sobolev embedding. $f: \bar{\Omega} \times \mathbb{R} \rightarrow \mathbb{R}, g: \mathbb{R}^{+} \rightarrow \mathbb{R}^{+}$are continuous functions that satisfy the following conditions:

(G1) There exists $\alpha_{0}>0$ such that $g(t) \geq \alpha_{0}$ for all $t \geq 0$.

(G2) There exists $\sigma$ satisfied $1<\frac{p}{\sigma}<p^{*}$ and $G(t) \geq \sigma g(t) t$ for all $t \geq 0$, where

$$
G(t)=\int_{0}^{t} g(s) d s .
$$

(H1) $h(x, u) \in C(\Omega \times \mathbb{R}, \mathbb{R}), h(x,-u)=-h(x, u)$ for all $u \in \mathbb{R}$.

(H2) $\lim _{|u| \rightarrow \infty} \frac{h(x, u)}{|u|^{p^{*}-1}}=0$ uniformly for $x \in \Omega$.

(H3) $\lim _{|u| \rightarrow 0^{+}} \frac{h(x, u)}{u^{p / \sigma-1}}=\infty$ uniformly for $x \in \Omega$.

Much interest has grown on problems involving critical exponents, starting from the celebrated paper by Brezis and Nirenberg [1]. For example, Li and Zou [2] obtained infinitely many solutions with odd nonlinearity. Chen and Li [3] obtained the existence of infinitely many solutions by using the minimax procedure. For more related results, we refer the interested reader to [4-9] and references therein.

(c) 2015 Zhou and Song. This article is distributed under the terms of the Creative Commons Attribution 4.0 International License (http://creativecommons.org/licenses/by/4.0/), which permits unrestricted use, distribution, and reproduction in any medium, provided you give appropriate credit to the original author(s) and the source, provide a link to the Creative Commons license, and indicate if changes were made. 
On the one hand, for the special case of problem (1.1), equation (1.1) reduces to the following Dirichlet problem of Kirchhoff type:

$$
\left\{\begin{array}{l}
-\left(a+b \int_{\Omega}|\nabla u|^{2} d x\right) \Delta u=f(x, u), \quad x \in \Omega, \\
\left.u\right|_{\partial \Omega}=0
\end{array}\right.
$$

where $\Omega \subset \mathbb{R}^{N}$, problem (1.2) is a generalization of a model introduced by Kirchhoff [10]. More precisely, Kirchhoff proposed a model given by the equation

$$
\rho \frac{\partial^{2} u}{\partial t^{2}}-\left(\frac{\rho_{0}}{h}+\frac{E}{2 L} \int_{0}^{L}\left|\frac{\partial u}{\partial x}\right|^{2} d x\right) \frac{\partial^{2} u}{\partial x^{2}}=0
$$

where $\rho, \rho_{0}, h, E, L$ are constants, which extends the classical d'Alembert wave equation, by considering the effects of the changes in the length of the strings during the vibrations. The equation (1.2) is related to the stationary analog of problem (1.3). Equation (1.2) received much attention only after Lions [11] proposed an abstract framework to the problem. Some important and interesting results can be found; see for example [12-19]. We note that results dealing with the problem (1.2) with critical nonlinearity are relatively scarce.

In [20], by means of a direct variational method, the authors proved the existence and multiplicity of solutions to a class of $p$-Kirchhoff-type problem with Dirichlet boundary data. In [21], the author showed the existence of infinite solutions to the $p$-Kirchhoff-type quasilinear elliptic equation. But they did not give any further information on the sequence of solutions. Recently, Kajikiya [22] established a critical point theorem related to the symmetric mountain-pass lemma and applied to a sublinear elliptic equation. However, there are no such results on Kirchhoff-type problems (1.1).

Motivated by reasons above, the aim of this paper is to show that the existence of infinitely many solutions of problem (1.1), and there exists a sequence of infinitely many arbitrarily small solutions converging to zero by using a new version of the symmetric mountain-pass lemma due to Kajikiya [22].

To the best of our knowledge, the existence and multiplicity of solutions to problem (1.1) has not ever been studied by variational methods. As we shall see in the present paper, problem (1.1) can be viewed as an elliptic equation coupled with a non-local term. The competing effect of the non-local term with the critical nonlinearity and the lack of compactness of the embedding of $H_{0}^{1, p}(\Omega)$ into the space $L^{p^{*}}(\Omega)$, prevents us from using the variational methods in a standard way. Some new estimates for such a Kirchhoff equation involving Palais-Smale sequences, which are key points to the application of this kind of theory, are needed to be re-established. We mainly follow the idea of [22, 23]. Let us point out that although the idea was used before for other problems, the adaptation to the procedure to our problem is not trivial at all, since the appearance of non-local term, we must consider our problem for suitable space and so we need more delicate estimates.

Our main result in this paper is the following.

Theorem 1.1 Suppose that (G1)-(G2), (H1)-(H3) hold. There then exists $\lambda^{*}>0$ such that, for any $\lambda \in\left(0, \lambda^{*}\right)$, problem (1.1) has a sequence of non-trivial solutions $\left\{u_{n}\right\}$ and $u_{n} \rightarrow 0$ as $n \rightarrow \infty$. 


\section{Preliminary lemmas}

We consider the energy functional $J: W_{0}^{1, p}(\Omega) \rightarrow \mathbb{R}$ defined by

$$
J(u)=\frac{1}{p} G\left(\|u\|^{p}\right)-\lambda \int_{\Omega} H(x, u) d x-\frac{1}{p^{*}} \int_{\Omega}|u|^{p^{*}} d x,
$$

where $W_{0}^{1, p}(\Omega)$ is the Sobolev space endowed with the norm $\|u\|^{p}=\int_{\Omega}|\nabla u|^{p} d x$. Standard arguments [24] show that a critical point of $J$ is a weak solution of problem (1.1). We try to use a new version of the symmetric mountain-pass lemma due to Kajikiya [22]. But since the functional $J(u)$ is not bounded from below, we could not use the theory directly. So we follow [23] to consider a truncated functional of $J(u)$. Denote by $J^{\prime}: E \rightarrow E^{*}$ the derivative operator of $J$ in the weak sense. Then

$$
\begin{aligned}
\left\langle J^{\prime}(u), v\right\rangle= & g\left(\|u\|^{p}\right) \int_{\Omega}\left(|\nabla u|^{p-2} \nabla u \cdot \nabla v\right) d x-\int_{\Omega}|u|^{p^{*}-2} u v d x \\
& -\lambda \int_{\Omega} h(x, u) v d x, \quad \forall u, v \in W_{0}^{1, p}(\Omega) .
\end{aligned}
$$

To use variational methods, we give some results related to the Palais-Smale compactness condition. Recall that a sequence $\left(u_{n}\right)$ is a Palais-Smale sequence of $J$ at the level $c$, if $J\left(u_{n}\right) \rightarrow c$ and $J^{\prime}\left(u_{n}\right) \rightarrow 0$.

We recall the second concentration-compactness principle of Lions $[25,26]$.

Lemma 2.1 $[25,26]$ Let $\left\{u_{n}\right\}$ be a weakly convergent sequence to $u$ in $W_{0}^{1, p}(\Omega)$ such that $\left|u_{n}\right|^{p^{*}} \rightarrow v$ and $\left|\nabla u_{n}\right| \rightarrow \mu$ in the sense of measures. Then, for some at most countable index set $I$,

(i) $v=|u|^{p^{*}}+\sum_{j \in I} \delta_{x_{j}} v_{j}, v_{j}>0$,

(ii) $\mu \geq|\nabla u|^{p}+\sum_{j \in I} \delta_{x_{j}} \mu_{j}, \mu_{j}>0$,

(iii) $\mu_{j} \geq S v_{j}^{p / p^{*}}$,

where $S$ is the best Sobolev constant, i.e. $S=\inf \left\{\int_{\mathbb{R}^{N}}|\nabla u|^{p} d x: \int_{\mathbb{R}^{N}}|u|^{p^{*}} d x=1\right\}, x_{j} \in \mathbb{R}^{N}, \delta_{x_{j}}$ are Dirac measures at $x_{j}$ and $\mu_{j}, v_{j}$ are constants.

Under assumptions (H1) and (H2), we have

$$
h(x, s) s=o\left(|s|^{p^{*}}\right), \quad H(x, s)=o\left(|s|^{p^{*}}\right)
$$

which means that, for all $\varepsilon>0$, there exist $a(\varepsilon), b(\varepsilon)>0$ such that

$$
\begin{aligned}
& |h(x, s) s| \leq a(\varepsilon)+\varepsilon|s|^{p^{*}}, \\
& |H(x, s)| \leq b(\varepsilon)+\varepsilon|s|^{p^{*}} .
\end{aligned}
$$

Hence,

$$
H(x, s)-\frac{\sigma}{p} h(x, s) s \leq c(\varepsilon)+\varepsilon|s|^{p^{*}}
$$

for some $c(\varepsilon)>0$. 
Lemma 2.2 Suppose that (G1)-(G2), (H1)-(H3) hold. Then, for any $\lambda>0$, the functional $J$ satisfies the local $(P S)_{c}$ condition in

$$
c \in\left(-\infty, \frac{p^{*} \sigma-p}{2 p p^{*}}\left(\alpha_{0} S\right)^{N / p}-\lambda c\left(\frac{p^{*} \sigma-p}{2 p p^{*} \lambda}\right)|\Omega|\right)
$$

in the following sense: if

$$
J\left(u_{n}\right) \rightarrow c<\frac{p^{*} \sigma-p}{2 p p^{*}}\left(\alpha_{0} S\right)^{N / p}-\lambda c\left(\frac{p^{*} \sigma-p}{2 p p^{*} \lambda}\right)|\Omega|
$$

and $J^{\prime}\left(u_{n}\right) \rightarrow 0$ for some sequence in $W_{0}^{1, p}(\Omega)$, then $\left\{u_{n}\right\}$ contains a subsequence converging strongly in $W_{0}^{1, p}(\Omega)$.

Proof Let $\left\{u_{n}\right\}$ be a sequence in $W_{0}^{1, p}(\Omega)$ such that

$$
\begin{aligned}
J\left(u_{n}\right)=\frac{1}{p} G\left(\left\|u_{n}\right\|^{p}\right)-\lambda \int_{\Omega} H\left(x, u_{n}\right) d x-\frac{1}{p^{*}} \int_{\Omega}\left|u_{n}\right|^{p^{*}} d x=c+o(1), \\
\begin{aligned}
\left\langle J^{\prime}\left(u_{n}\right), v\right\rangle= & g\left(\left\|u_{n}\right\|^{p}\right) \int_{\Omega}\left(\left|\nabla u_{n}\right|^{p-2} \nabla u_{n} \cdot \nabla v\right) d x-\int_{\Omega}\left|u_{n}\right|^{p^{*}-2} u_{n} v d x \\
& -\lambda \int_{\Omega} h\left(x, u_{n}\right) v d x=o(1)\left\|u_{n}\right\| .
\end{aligned}
\end{aligned}
$$

By (2.4) and (2.5), we have

$$
\begin{aligned}
c+o(1)\left\|u_{n}\right\|= & J\left(u_{n}\right)-\frac{\sigma}{p}\left\langle J^{\prime}\left(u_{n}\right), u_{n}\right\rangle \\
= & \frac{1}{p} G\left(\left\|u_{n}\right\|^{p}\right)-\frac{\sigma}{p} g\left(\left\|u_{n}\right\|^{p}\right)\left\|u_{n}\right\|^{p}+\left(\frac{\sigma}{p}-\frac{1}{p^{*}}\right) \int_{\Omega}\left|u_{n}\right|^{p^{*}} d x \\
& -\lambda \int_{\Omega} H\left(x, u_{n}\right) d x+\frac{\sigma}{p} \lambda \int_{\Omega} h\left(x, u_{n}\right) u_{n} d x \\
\geq & \frac{p^{*} \sigma-p}{p p^{*}} \int_{\Omega}\left|u_{n}\right|^{p^{*}} d x-\lambda \int_{\Omega} H\left(x, u_{n}\right) d x \\
& +\frac{\sigma}{p} \lambda \int_{\Omega} h\left(x, u_{n}\right) u_{n} d x,
\end{aligned}
$$

i.e.

$$
\frac{p^{*} \sigma-p}{p p^{*}} \int_{\Omega}\left|u_{n}\right|^{p^{*}} d x \leq \lambda \int_{\Omega}\left(H\left(x, u_{n}\right)-\frac{\sigma}{p} h\left(x, u_{n}\right) u_{n}\right) d x+c+o(1)\left\|u_{n}\right\| .
$$

Then by (2.3), we have

$$
\left(\frac{p^{*} \sigma-p}{p p^{*}}-\lambda \varepsilon\right) \int_{\Omega}\left|u_{n}\right|^{p^{*}} d x \leq \lambda c(\varepsilon)|\Omega|+c+o(1)\left\|u_{n}\right\| .
$$

Setting $\varepsilon=\frac{p^{*} \sigma-p}{2 p p^{*} \lambda}$, we get

$$
\int_{\Omega}\left|u_{n}\right|^{p^{*}} d x \leq M+o(1)\left\|v_{n}\right\|
$$


where $o(1) \rightarrow 0$ and $M$ is a some positive number. On the other hand, by (2.2) and (2.6), we have

$$
\begin{aligned}
c+o(1)\left\|u_{n}\right\| & =J\left(u_{n}\right) \\
& =\frac{1}{p} G\left(\left\|u_{n}\right\|^{p}\right)-\lambda \int_{\Omega} H\left(x, u_{n}\right) d x-\frac{1}{p^{*}} \int_{\Omega}\left|u_{n}\right|^{p^{*}} d x \\
& \geq \frac{\alpha_{0} \sigma}{p}\left\|u_{n}\right\|^{p}-\lambda b(\varepsilon)|\Omega|-\left[\frac{1}{p^{*}}+\lambda \varepsilon\right] \int_{\Omega}\left|u_{n}\right|^{p^{*}} d x .
\end{aligned}
$$

Therefore, the inequalities (2.6) and (2.7) imply that $\left\{u_{n}\right\}$ is bounded in $W_{0}^{1, p}(\Omega)$. Hence, up to a subsequence, we may assume that

$$
\begin{aligned}
& u_{n} \rightarrow u \quad \text { weakly in } W_{0}^{1, p}(\Omega), \\
& u_{n} \rightarrow u \quad \text { a.e. in } \Omega, \\
& u_{n} \rightarrow u \quad \text { in } L^{s}(\Omega), 1 \leq s<p^{*}, \\
& \left|\nabla u_{n}\right|^{p} \rightarrow \mu \quad\left(\text { weak }^{*}\right. \text {-sense of measures), } \\
& \left|u_{n}\right|^{p^{*}} \rightarrow v \quad \text { (weak*-sense of measures), }
\end{aligned}
$$

where $\mu$ and $v$ are a nonnegative bounded measures on $\bar{\Omega}$. Then, by the concentrationcompactness principle due to Lions $[25,26]$, there exists some at most countable index set $I$ such that

$$
\begin{aligned}
& v=|u|^{p^{\star}}+\sum_{j \in I} v_{j} \delta_{x_{j}}, \quad v_{j}>0, \\
& \mu \geq|\nabla u|^{p}+\sum_{j \in I} \mu_{j} \delta_{x_{j}}, \quad \mu_{j}>0, \\
& S v_{j}^{p / p^{\star}} \leq \mu_{j},
\end{aligned}
$$

where $\delta_{x_{j}}$ is the Dirac measure mass at $x_{j} \in \bar{\Omega}$. Let $\psi(x) \in C_{0}^{\infty}$ such that $0 \leq \psi \leq 1$,

$$
\psi(x)= \begin{cases}1 & \text { if }|x|<1 \\ 0 & \text { if }|x| \geq 2\end{cases}
$$

and $|\nabla \psi|_{\infty} \leq 2$

For $\varepsilon>0$ and $j \in I$, denote $\psi_{\varepsilon}^{j}(x)=\psi\left(\left(x-x_{j}\right) / \varepsilon\right)$. Since $J^{\prime}\left(u_{n}\right) \rightarrow 0$ and $\left(\psi_{\varepsilon}^{j} u_{n}\right)$ is bounded, $\left\langle J^{\prime}\left(u_{n}\right), \psi_{\varepsilon}^{j} u_{n}\right\rangle \rightarrow 0$ as $n \rightarrow \infty$; that is,

$$
\begin{aligned}
g\left(\left\|u_{n}\right\|^{p}\right) \int_{\Omega}\left|\nabla u_{n}\right|^{p} \psi_{\varepsilon}^{j} d x & \\
= & -g\left(\left\|u_{n}\right\|^{p}\right) \int_{\Omega} u_{n}\left|\nabla u_{n}\right|^{p-2} \nabla u_{n} \nabla \psi_{\varepsilon}^{j} d x \\
& +\lambda \int_{\Omega} h\left(x, u_{n}\right) u_{n} \psi_{\varepsilon}^{j} d x+\int_{\Omega}\left|u_{n}\right|^{p^{*}} \psi_{\varepsilon}^{j} d x+o_{n}(1) .
\end{aligned}
$$


By (2.8) and Vitali's theorem, we see that

$$
\lim _{n \rightarrow \infty} \int_{\Omega}\left|u_{n} \nabla \psi_{\varepsilon}^{j}\right|^{p} d x=\int_{\Omega}\left|u \nabla \psi_{\varepsilon}^{j}\right|^{p} d x
$$

Hence, by Hölder's inequality we obtain

$$
\begin{aligned}
& \left.\limsup _{n \rightarrow \infty}\left|\int_{\Omega} u_{n}\right| \nabla u_{n}\right|^{p-2} \nabla u_{n} \nabla \psi_{\varepsilon}^{j} d x \mid \\
& \leq \limsup _{n \rightarrow \infty}\left(\int_{\Omega}\left|\nabla u_{n}\right|^{p} d x\right)^{(p-1) / p}\left(\int_{\Omega}\left|u_{n} \nabla \psi_{\varepsilon}^{j}\right|^{p} d x\right)^{1 / p} \\
& \leq C_{1}\left(\int_{B\left(x_{j}, 2 \varepsilon\right)}|u|^{p}\left|\nabla \psi_{\varepsilon}^{j}\right|^{p} d x\right)^{1 / p} \\
& \leq C_{1}\left(\int_{B\left(x_{j}, 2 \varepsilon\right)}\left|\nabla \psi_{\varepsilon}^{j}\right|^{N} d x\right)^{1 / N}\left(\int_{B\left(x_{j}, 2 \varepsilon\right)}|u|^{p^{*}} d x\right)^{1 / p^{*}} \\
& \quad \leq C_{2}\left(\int_{B\left(x_{j}, 2 \varepsilon\right)}|u|^{p^{*}} d x\right)^{1 / p^{*}} \rightarrow 0 \text { as } \varepsilon \rightarrow 0 .
\end{aligned}
$$

Since $\psi_{\varepsilon}^{j}$ has compact support, letting $n \rightarrow \infty$ in (2.10) we deduce from (2.10) and (2.11) that

$$
\alpha_{0} \int_{\Omega} \psi_{\varepsilon}^{j} d \mu \leq C_{2}\left(\int_{B\left(x_{j}, 2 \varepsilon\right)}|u|^{p^{*}} d x\right)^{1 / p^{\star}}+\lambda \int_{B\left(x_{j}, 2 \varepsilon\right)} f(x, u) u d x+\int_{\Omega} \psi_{\varepsilon}^{j} d \nu
$$

Letting $\varepsilon \rightarrow 0$, we obtain $\alpha_{0} \mu_{j} \leq v_{j}$. Therefore,

$$
\left(\alpha_{0} S\right)^{N / p} \leq v_{j}
$$

We will prove that this inequality is not possible. Let us assume that $\left(\alpha_{0} S\right)^{N / p} \leq v_{j_{0}}$ for some $j_{0} \in I$. From (G2) we see that

$$
G\left(\left\|u_{n}\right\|^{p}\right)-\sigma g\left(\left\|u_{n}\right\|^{p}\right)\left\|u_{n}\right\|^{p} \geq 0 \quad \text { for all } n \text {. }
$$

Since

$$
c=J\left(u_{n}\right)-\frac{\sigma}{p}\left\langle J^{\prime}\left(u_{n}\right), u_{n}\right\rangle+o_{n}(1),
$$

it follows that

$$
\begin{aligned}
c & =\lim _{n \rightarrow \infty}\left(J\left(u_{n}\right)-\frac{\sigma}{p}\left\langle J^{\prime}\left(u_{n}\right), u_{n}\right\rangle\right) \\
& \geq\left(\frac{\sigma}{p}-\frac{1}{p^{*}}\right) \int_{\Omega}\left|u_{n}\right|^{p^{*}} d x-\lambda \int_{\Omega}\left[H\left(x, u_{n}\right)-\frac{\sigma}{p} h\left(x, u_{n}\right) u_{n}\right] d x \\
& \geq\left(\frac{p^{*} \sigma-p}{p p^{*}}-\lambda \varepsilon\right) \int_{\Omega} \psi_{\varepsilon}^{j_{0}}\left|u_{n}\right|^{p^{*}} d x-\lambda c(\varepsilon)|\Omega| .
\end{aligned}
$$


Letting $\varepsilon=\frac{p^{*} \sigma-p}{2 p p^{*} \lambda}$ and $n \rightarrow \infty$, we obtain

$$
\begin{aligned}
c & \geq \frac{p^{*} \sigma-p}{2 p p^{*}} \sum_{j \in J} \psi_{\varepsilon}^{j_{0}}\left(x_{j}\right) v_{j}-\lambda c\left(\frac{p^{*} \sigma-p}{2 p p^{*} \lambda}\right)|\Omega| \\
& \geq \frac{p^{*} \sigma-p}{2 p p^{*}}\left(\alpha_{0} S\right)^{N / p}-\lambda c\left(\frac{p^{*} \sigma-p}{2 p p^{*} \lambda}\right)|\Omega| .
\end{aligned}
$$

This is impossible. Then $I=\emptyset$, and hence $u_{n} \rightarrow u$ in $L^{p^{*}}(\Omega)$.

Then, using (2.8) and the fact that $u_{n} \rightarrow u$ in $L^{p^{*}}(\Omega)$, we have

$$
\lim _{n \rightarrow \infty} \int_{\Omega} h\left(x, u_{n}\right)\left(u_{n}-u\right) d x=0
$$

and

$$
\lim _{n \rightarrow \infty} \int_{\Omega}\left|u_{n}\right|^{p^{*}-2} u_{n}\left(u_{n}-u\right) d x=0
$$

From $\left\langle J^{\prime}\left(u_{n}\right), u_{n}-u\right\rangle=o_{n}(1)$, we deduce that

$$
\begin{aligned}
\left\langle J^{\prime}\left(u_{n}\right), u_{n}-u\right\rangle= & g\left(\left\|u_{n}\right\|^{p}\right) \int_{\Omega}\left|\nabla u_{n}\right|^{p-2} \nabla u_{n} \nabla\left(u_{n}-u\right) d x \\
& -\lambda \int_{\Omega} h\left(x, u_{n}\right)\left(u_{n}-u\right) d x-\int_{\Omega}\left|u_{n}\right|^{p^{*}-2} u_{n}\left(u_{n}-u\right) d x=o_{n}(1) .
\end{aligned}
$$

This, (2.13), and (2.14) imply

$$
\lim _{n \rightarrow \infty} g\left(\left\|u_{n}\right\|^{p}\right) \int_{\Omega}\left|\nabla u_{n}\right|^{p-2} \nabla u_{n} \nabla\left(u_{n}-u\right) d x=0
$$

Since $u_{n}$ is bounded and $g$ is continuous, up to subsequence, there is $t_{0} \geq 0$ such that

$$
g\left(\left\|u_{n}\right\|^{p}\right) \rightarrow g\left(t_{0}^{p}\right) \geq \alpha_{0} \quad \text { as } n \rightarrow \infty
$$

and so

$$
\lim _{n \rightarrow \infty} \int_{\Omega}\left|\nabla u_{n}\right|^{p-2} \nabla u_{n} \nabla\left(u_{n}-u\right) d x=0 .
$$

Thus by the $\left(S_{+}\right)$property, $u_{n} \rightarrow u$ strongly in $W_{0}^{1, p}(\Omega)$. The proof is complete.

\section{Existence of a sequence of arbitrarily small solutions}

In this section, we prove the existence of infinitely many solutions of (1.1) which tend to zero. Let $X$ be a Banach space and denote

$$
\Sigma:=\{A \subset X \backslash\{0\}: A \text { is closed in } X \text { and symmetric with respect to the origin }\} .
$$

For $A \in \Sigma$, we define genus $\gamma(A)$ as

$$
\gamma(A):=\inf \left\{m \in N: \exists \varphi \in C\left(A, \mathbb{R}^{m} \backslash\{0\},-\varphi(x)=\varphi(-x)\right)\right\}
$$


If there is no mapping $\varphi$ as above for any $m \in N$, then $\gamma(A)=+\infty$. Let $\Sigma_{k}$ denote the family of closed symmetric subsets $A$ of $X$ such that $0 \notin A$ and $\gamma(A) \geq k$. We list some properties of the genus (see [22, 27]).

Proposition 3.1 Let $A$ and $B$ be closed symmetric subsets of $X$ which do not contain the origin. Then the following hold.

(1) If there exists an odd continuous mapping from $A$ to $B$, then $\gamma(A) \leq \gamma(B)$.

(2) If there is an odd homeomorphism from $A$ to $B$, then $\gamma(A)=\gamma(B)$.

(3) If $\gamma(B)<\infty$, then $\gamma \overline{(A \backslash B)} \geq \gamma(A)-\gamma(B)$.

(4) Then $n$-dimensional sphere $S^{n}$ has a genus of $n+1$ by the Borsuk-Ulam theorem.

(5) If $A$ is compact, then $\gamma(A)<+\infty$ and there exists $\delta>0$ such that $U_{\delta}(A) \in \Sigma$ and $\gamma\left(U_{\delta}(A)\right)=\gamma(A)$, where $U_{\delta}(A)=\{x \in X:\|x-A\| \leq \delta\}$.

The following version of the symmetric mountain-pass lemma is due to Kajikiya [22].

Lemma 3.1 Let $E$ be an infinite-dimensional space and $J \in C^{1}(E, R)$ and suppose the following conditions hold.

$\left(C_{1}\right) J(u)$ is even, bounded from below, $J(0)=0$ and $J(u)$ satisfies the local Palais-Smale condition, i.e. for some $\bar{c}>0$, in the case when every sequence $\left\{u_{k}\right\}$ in E satisfying $\lim _{k \rightarrow \infty} J\left(u_{k}\right)=c<\bar{c}$ and $\lim _{k \rightarrow \infty}\left\|J^{\prime}\left(u_{k}\right)\right\|_{E^{*}}=0$ has a convergent subsequence.

$\left(C_{2}\right)$ For each $k \in N$, there exists an $A_{k} \in \Sigma_{k}$ such that $\sup _{u \in A_{k}} J(u)<0$.

Then either $\left(\mathrm{R}_{1}\right)$ or $\left(\mathrm{R}_{2}\right)$ below holds.

$\left(R_{1}\right)$ There exists a sequence $\left\{u_{k}\right\}$ such that $J^{\prime}\left(u_{k}\right)=0, J^{\prime}\left(u_{k}\right)<0$, and $\left\{u_{k}\right\}$ converges to zero.

$\left(\mathrm{R}_{2}\right)$ There exist two sequences $\left\{u_{k}\right\}$ and $\left\{v_{k}\right\}$ such that $J^{\prime}\left(u_{k}\right)=0, J\left(u_{k}\right)<0, u_{k} \neq 0$, $\lim _{k \rightarrow \infty} u_{k}=0, J^{\prime}\left(v_{k}\right)=0, J\left(v_{k}\right)<0, \lim _{k \rightarrow \infty} v_{k}=0$, and $\left\{v_{k}\right\}$ converges to a non-zero limit.

Remark 3.1 From Lemma 3.1 we have a sequence $\left\{u_{k}\right\}$ of critical points such that $J\left(u_{k}\right) \leq$ $0, u_{k} \neq 0$ and $\lim _{k \rightarrow \infty} u_{k}=0$.

In order to get infinitely many solutions we need some lemmas. Let $\varepsilon=\frac{1}{p^{*} \lambda}$, from (2.4) we have

$$
\begin{aligned}
J(u) & :=\frac{1}{p} G\left(\|u\|^{p}\right)-\lambda \int_{\Omega} H(x, u) d x-\frac{1}{p^{*}} \int_{\Omega}|u|^{p^{*}} d x \\
& \geq \frac{\alpha_{0} \sigma}{p} \int_{\Omega}|\nabla u|^{p} d x-\left(\frac{1}{p^{*}}+\varepsilon \lambda\right) \int_{\Omega}|u|^{p^{*}} d x-\lambda b(\varepsilon)|\Omega| \\
& =\frac{\alpha_{0} \sigma}{p} \int_{\Omega}|\nabla u|^{p} d x-\frac{2}{p^{*}} \int_{\Omega}|u|^{p^{*}} d x-\lambda b\left(\frac{1}{p^{*} \lambda}\right)|\Omega| \\
& \geq L_{1}\|u\|^{p}-L_{2}\|u\|^{p^{*}}-L_{3} \lambda,
\end{aligned}
$$

where $L_{1}, L_{2}, L_{3}$ are some positive constants.

Let $Q(t)=L_{1} t^{p}-L_{2} t^{p^{*}}-L_{3} \lambda$. Then

$$
J(u) \geq Q(\|u\|) .
$$


Furthermore, there exists $\lambda_{*}:=\frac{p L_{1}}{N L_{3}}\left(\frac{p L_{1}}{p^{*} L_{2}}\right)^{(N-p) / p}$ such that for $\lambda \in\left(0, \lambda_{*}\right), Q(t)$ attains its positive maximum, that is, there exists

$$
R_{1}=\left(\frac{p L_{1}}{p^{*} L_{2}}\right)^{(N-p) / p^{2}}
$$

such that

$$
e_{1}=Q\left(R_{1}\right)=\max _{t \geq 0} Q(t)>0
$$

Therefore, for $e_{0} \in\left(0, e_{1}\right)$, we may find $R_{0}<R_{1}$ such that $Q\left(R_{0}\right)=e_{0}$. Now we define

$$
\chi(t)= \begin{cases}1, & 0 \leq t \leq R_{0}, \\ \frac{L_{1} t^{p}-\lambda L_{3}-e_{1}}{L_{2} t^{p^{*}}}, & t \geq R_{1}, \\ C^{\infty}, \quad \chi(t) \in[0,1], & R_{0} \leq t \leq R_{1} .\end{cases}
$$

Then it is easy to see $\chi(t) \in[0,1]$ and $\chi(t)$ is $C^{\infty}$. Let $\varphi(u)=\chi(\|u\|)$ and consider the perturbation of $J(u)$ :

$$
G(u):=\frac{1}{p} G\left(\|u\|^{p}\right)-\frac{1}{p^{*}} \varphi(u) \int_{\Omega}|u|^{p^{*}} d x-\lambda \varphi(u) \int_{\Omega} H(x, u) d x .
$$

Then

$$
G(u) \geq L_{1}\|u\|^{p}-L_{2} \varphi(v)\|u\|^{p^{*}}-L_{3} \lambda=\bar{Q}(\|u\|),
$$

where $\bar{Q}(t)=L_{1} t^{p}-L_{2} \chi(t) t^{p^{*}}-L_{3} \lambda$ and

$$
\bar{Q}(t)= \begin{cases}Q(t), & 0 \leq t \leq R_{0} \\ e_{1}, & t \geq R_{1}\end{cases}
$$

From the above arguments, we have the following.

Lemma 3.2 Let $G(u)$ is defined as in (3.1). Then:

(i) $G \in C^{1}\left(W_{0}^{1, p}(\Omega), \mathbb{R}\right)$ and $G$ is even and bounded from below.

(ii) If $G(u)<e_{0}$, then $\bar{Q}(\|u\|)<e_{0}$, consequently, $\|u\|<R_{0}$ and $J(u)=G(u)$.

(iii) There exists $\lambda^{*}$ such that, for $\lambda \in\left(0, \lambda^{*}\right)$, G satisfies a local $(P S)_{c}$ condition for

$$
c<e_{0} \in\left(0, \min \left\{e_{1}, \frac{p^{*} \sigma-p}{2 p p^{*}}\left(\alpha_{0} S\right)^{N / p}-\lambda c\left(\frac{p^{*} \sigma-p}{2 p p^{*} \lambda}\right)|\Omega|\right\}\right) .
$$

Lemma 3.3 Suppose that (G1)-(G2), (H3) hold. Then, for any $k \in \mathbb{N}$, there exists $\delta=\delta(k)>$ 0 such that $\gamma\left(\left\{u \in W_{0}^{1, p}(\Omega): G(u) \leq-\delta(k)\right\} \backslash\{0\}\right) \geq k$.

Proof First of all, by (H3) of Theorem 1.1, for any fixed $u \in W_{0}^{1, p}(\Omega), u \neq 0$, we have

$$
H(x, \rho u) \geq M(\rho)(\rho u)^{\frac{p}{\sigma}} \quad \text { with } M(\rho) \rightarrow \infty \text { as } \rho \rightarrow 0 .
$$


On the other hand, by integrating (G2), we obtain

$$
G(t) \leq \frac{G\left(t_{0}\right)}{t_{0}^{1 / \sigma}} t^{1 / \sigma}=C_{0} t^{1 / \sigma} \quad \text { for all } t \geq t_{0}>0
$$

Second, given any $k \in N$, let $E_{k}$ be a $k$-dimensional subspace of $W_{0}^{1, p}(\Omega)$. There then exists a positive constant $\delta$ such that

$$
\|u\| \leq \delta|u|_{p / \sigma} \quad \text { for all } u \in E_{k} .
$$

Therefore, for any $u \in E_{k}$ with $\|u\|=1$ and $\rho$ small enough, by (3.2) and (H3) we have

$$
\begin{aligned}
G(\rho u) & =\frac{1}{p} G\left(\|u\|^{p}\right)-\frac{1}{p^{*}} \varphi(u) \int_{\Omega}|u|^{p^{*}} d x-\lambda \varphi(u) \int_{\Omega} H(x, u) d x \\
& \leq \frac{C_{0}}{p} \rho^{\frac{p}{\sigma}}-\frac{\lambda M(\rho)}{\delta^{p / \sigma}} \rho^{\frac{p}{\sigma}} \\
& \leq\left(\frac{C_{0}}{p}-\frac{\lambda M(\rho)}{\delta^{p / \sigma}}\right) \rho^{\frac{p}{\sigma}} \\
& =-\delta(k)<0,
\end{aligned}
$$

since $\lim _{|\rho| \rightarrow 0} M(\rho)=+\infty$. That is,

$$
\left\{u \in E_{k}:\|u\|=\rho\right\} \subset\left\{u \in W_{0}^{1, p}(\Omega): G(u) \leq-\delta(k)\right\} \backslash\{0\} .
$$

This completes the proof.

Now we give the proof of Theorem 1.1 as follows.

Proof of Theorem 1.1 Recall that

$$
\Sigma_{k}=\left\{A \in W_{0}^{1, p}(\Omega) \backslash\{0\}: A \text { is closed and } A=-A, \gamma(A) \geq k\right\}
$$

and define

$$
c_{k}=\inf _{A \in \Sigma_{k}} \sup _{u \in A} G(u)
$$

By Lemma 3.2(i) and Lemma 3.3, we know that $-\infty<c_{k}<0$. Therefore, assumptions $\left(C_{1}\right)$ and $\left(C_{2}\right)$ of Lemma 3.1 are satisfied. This means that $G$ has a sequence of solutions $\left\{u_{n}\right\}$ converging to zero. Hence, Theorem 1.1 follows by Lemma 3.2(ii).

\section{A special case of problem (1.1)}

We consider the following special case of problem (1.1):

$$
\begin{aligned}
& -\left(\alpha+\beta \int_{\Omega}|\nabla u|^{p} d x\right) \Delta_{p} u=\lambda f(x, u)+|u|^{p^{*}-2} u \text { in } \Omega, \\
& u=0 \quad \text { on } \partial \Omega,
\end{aligned}
$$


where $\Omega$ is a bounded smooth domain of $\mathbb{R}^{N}, 1<p<N<2 p, \alpha$ and $\beta$ are positive constants.

$$
\text { Set } g(t)=\alpha+\beta t \text {. Then } g(t) \geq \alpha \text { and }
$$

$$
G(t)=\int_{0}^{1} g(s) d s=\alpha t+\frac{1}{2} \beta t^{2} \geq \frac{1}{2}(\alpha+\beta t) t=\sigma g(t) t
$$

where $\sigma=1 / 2$. Hence the conditions (G1) and (G2) are satisfied.

For this case, a typical example of a function satisfying the conditions (F1)-(F3) is given by

$$
f(x, t)=\sum_{i=1}^{k} a_{i}(x)|t|^{q_{i}-2} t,
$$

where $k \geq 1,1<q_{i}<\frac{p}{\sigma}$, and $a_{i}(x) \in C(\bar{\Omega})$. In view of Theorem 1.1, we have the following corollary.

Corollary 4.1 Suppose that (F1)-(F3) hold. There then exists $\lambda^{*}>0$ such that for any $\lambda \in$ $\left(0, \lambda^{*}\right)$, problem (4.1) has a sequence of non-trivial solutions $\left\{u_{n}\right\}$ and $u_{n} \rightarrow 0$ as $n \rightarrow \infty$.

Competing interests

The authors declare that they have no competing interests.

\section{Authors' contributions}

CZ carried out the theoretical studies, and participated in the sequence alignment and drafted the manuscript. YS participated in the design of the study and performed the statistical analysis. All authors read and approved the final manuscript.

\section{Author details}

${ }^{1}$ College of Mathematics, Changchun Normal University, Changchun, Jilin 130032, P.R. China. ${ }^{2}$ Scientific Research Department, Changchun Normal University, Changchun, Jilin 130032, P.R. China.

\section{Acknowledgements}

The authors are supported by NSFC (Grant No. 11301038).

Received: 21 June 2015 Accepted: 19 November 2015 Published online: 02 December 2015

\section{References}

1. Brezis, H, Nirenberg, L: Positive solutions of nonlinear elliptic equations involving critical exponents. Commun. Pure Appl. Math. 34, 437-477 (1983)

2. Li, S, Zou, W: Remarks on a class of elliptic problems with critical exponents. Nonlinear Anal. 32, 769-774 (1998)

3. Chen, J, Li, S: On multiple solutions of a singular quasi-linear equation on unbounded domain. J. Math. Anal. Appl. 275, 733-746 (2002)

4. Chabrowski, J: On multiple solutions for the nonhomogeneous $p$-Laplacian with a critical Sobolev exponent. Differ. Integral Equ. 8, 705-716 (1995)

5. Ferrero, A, Gazzola, F: Existence of solutions for singular critical growth semilinear elliptic equations. J. Differ. Equ. 177, 494-522 (2001)

6. Garcia Azorero, J, Peral, I: Hardy inequalities and some critical elliptic and parabolic problems. J. Differ. Equ. 144, 441-476 (1998)

7. Ghoussoub, N, Yuan, C: Multiple solutions for quasi-linear PDEs involving the critical Sobolev and Hardy exponents. Trans. Am. Math. Soc. 352, 5703-5743 (2000)

8. He, XM, Zou, WM: Infinitely many arbitrarily small solutions for singular elliptic problems with critical Sobolev-Hardy exponents. Proc. Edinb. Math. Soc. 52, 97-108 (2009)

9. Silva, EA, Xavier, MS: Multiplicity of solutions for quasilinear elliptic problems involving critical Sobolev exponents Ann. Inst. Henri Poincaré, Anal. Non Linéaire 20, 341-358 (2003)

10. Kirchhoff, G: Mechanik. Teubner, Leipzig (1883)

11. Lions, JL: On some equations in boundary value problems of mathematical physics. In: Contemporary Developments in Continuum Mechanics and Partial Differential Equations. Proc. Internat. Sympos., Inst. Mat. Univ. Fed. Rio de Janeiro, Rio de Janeiro, 1977. North-Holland Math. Stud., vol. 30, pp. 284-346. North-Holland, Amsterdam (1978)

12. Corrêa, FJSA, Nascimento, RG: On a nonlocal elliptic system of $p$-Kirchhoff-type under Neumann boundary condition Math. Comput. Model. 49, 598-604 (2009) 
13. Corrêa, FJSA, Figueiredo, GM: On a elliptic equation of $p$-Kirchhoff type via variational methods. Bull. Aust. Math. Soc. 74, 263-277 (2006)

14. Hamydy, A, Massar, M, Tsouli, N: Existence of solutions for $p$-Kirchhoff type problems with critical exponent. Electron. J. Differ. Equ. 2011, 105 (2011)

15. He, X, Zou, W: Infinitely many positive solutions for Kirchhoff-type problems. Nonlinear Anal. 70, 1407-1414 (2009)

16. Ma, TF, Munoz Rivera, JE: Positive solutions for a nonlinear nonlocal elliptic transmission problem. Appl. Math. Lett. 16, 243-248 (2003)

17. Perera, K, Zhang, Z: Nontrivial solutions of Kirchhoff-type problems via the Yang index. J. Differ. Equ. 221, 246-255 (2006)

18. He, X, Zou, W: Multiplicity of solutions for a class of Kirchhoff type problems. Acta Math. Appl. Sin. 26, 387-394 (2010)

19. $\mathrm{Wu}, \mathrm{X}$ : Existence of nontrivial solutions and high energy solutions for Schrödinger-Kirchhoff-type equations in $\mathbb{R}^{N}$ Nonlinear Anal., Real World Appl. 12, 1278-1287 (2011)

20. Dai, GW, Hao, RF: Existence of solutions for a p(x)-Kirchhoff-type equation. J. Math. Anal. Appl. 359, 275-284 (2009)

21. Liu, DC: On a $p$-Kirchhoff equation via fountain theorem and dual fountain theorem. Nonlinear Anal. 72, 302-308 (2010)

22. Kajikiya, R: A critical-point theorem related to the symmetric mountain-pass lemma and its applications to elliptic equations. J. Funct. Anal. 225, 352-370 (2005)

23. Garcia Azorero, J, Peral, I: Multiplicity of solutions for elliptic problems with critical exponent or with a nonsymmetric term. Trans. Am. Math. Soc. 323, 877-895 (1991)

24. Willem, M: Minimax Theorems. Birkhäuser Boston, Boston (1996)

25. Lions, PL: The concentration compactness principle in the calculus of variations. The locally compact case, part I. Ann. Inst. Henri Poincaré, Anal. Non Linéaire 1, 109-145 (1984)

26. Lions, PL: The concentration compactness principle in the calculus of variations. The locally compact case, part II. Ann. Inst. Henri Poincaré, Anal. Non Linéaire 1, 223-283 (1984)

27. Rabinowitz, PH: Minimax Methods in Critical-Point Theory with Applications to Differential Equations. CBME Regional Conference Series in Mathematics, vol. 65. Am. Math. Soc., Providence (1986)

\section{Submit your manuscript to a SpringerOpen ${ }^{\ominus}$ journal and benefit from:}

- Convenient online submission

Rigorous peer review

- Immediate publication on acceptance

- Open access: articles freely available online

- High visibility within the field

- Retaining the copyright to your article 